\title{
A retrospective study of sepsis-associated encephalopathy: epidemiology, clinical features and adverse outcomes
}

\author{
Jiayi Chen ${ }^{1}$, Xiaobei Shi ${ }^{2}$, Mengyuan Diao ${ }^{1}$, Guangyong Jin ${ }^{1}$, Ying Zhu' ${ }^{1}$, Wei Hu ${ }^{1 *}$ and Shaosong $\mathrm{Xi}^{1{ }^{*}}$ (D)
}

\begin{abstract}
Background: Sepsis-associated encephalopathy (SAE) is a common complication of sepsis that may result in worse outcomes. This study was designed to determine the epidemiology, clinical features, and risk factors of SAE.

Methods: This was a retrospective study of all patients with sepsis who were admitted to the Critical Care Medicine Department of Hangzhou First People's Hospital Affiliated with Zhejiang University School of Medicine from January 2015 to December 2019.

Results: A total of 291 sepsis patients were screened, and 127 (43.6\%) were diagnosed with SAE. There were significant differences in median age, proportion of underlying diseases such as hypertension, Sequential Organ Failure Assessment (SOFA) score, Acute Physiology and Chronic Health Evaluation II (APACHE II) score, gastrointestinal infections, detection rate of Enterococcus, and 28-day mortality between the SAE and non-SAE groups. Both the SOFA score and APACHE II score were independent risk factors for SAE in patients with sepsis. All 127 SAE patients were divided into survival and non-survival groups. The age, SOFA score, and APACHE II score were independently associated with 28-day mortality in SAE patients.

Conclusion: In the present retrospective study, nearly half of patients with sepsis developed SAE, which was closely related to poor outcomes. Both the SOFA score and APACHE II score were independent risk factors for predicting the occurrence and adverse outcome of SAE.
\end{abstract}

Keywords: Sepsis, Sepsis-associated encephalopathy, Retrospective study

\section{Background}

Sepsis-associated encephalopathy (SAE) is a common complication of patients with sepsis and can manifest as mild disturbance of consciousness, disorientation, cognitive impairment, convulsion or deep coma [1, 2]. Importantly, SAE can result in dramatically poorer outcomes in patients with sepsis, with the mortality increasing with a rising SAE severity to a maximum of 70\% [3]. Surviving SAE patients are likely to exhibit prolonged or permanent side effects, including altered behaviour, cognitive impairment, reduced

\footnotetext{
*Correspondence: paolohu929@zju.edu.cn; simon52@sina.com 'Department of Critical Care Medicine, Affiliated Hangzhou First People's Hospital, Zhejiang University School of Medicine, No.261, Huansha Road, Zhejiang 310006, Hangzhou, China

Full list of author information is available at the end of the article
}

quality of life, or premature death [4]. To date, the diagnostic criteria and potential risk factors for SAE remain incompletely understood. Early identification, timely diagnosis and effective management may be important for the disease control in sepsis patients. The present study was therefore designed to explore the epidemiology, clinical characteristics and risk factors for SAE, as well as its adverse outcomes, via a retrospective study of 291 patients with sepsis.

\section{Methods}

\section{Patient selection}

This was a retrospective study of all patients with sepsis who were admitted to the Critical Care Medicine Department of Hangzhou First People's Hospital Affiliated 
with Zhejiang University School of Medicine from January 2015 to December 2019. Patient inclusion criteria were as follows: (1) Diagnosis of sepsis: Patients were diagnosed with sepsis based upon the sepsis 3.0 definition, which is the life-threatening organ dysfunction caused by the host's maladjusted response to infection [5]. (2) SAE identification: Cognitive and neuropsychiatric disorders clearly documented by medical staff (doctors and nurses), as well as a Glasgow coma score (GCS) $<15$ or manifestations of delirium (including inattention, disorientation, altered thinking, decreased psychomotor activity, and/or agitation) confirmed by the Confusion Assessment Method for the Intensive Care Unit (CAM-ICU). Patient exclusion criteria were sedative-related cognitive effects, primary central nervous system disease (cerebral vascular disease, central nervous system infection, autoimmune encephalitis, seizures), metabolic encephalopathy (hypoglycaemia, diabetic ketoacidosis, hepatic encephalopathy, pulmonary encephalopathy, uraemic encephalopathy), and toxicosis.

\section{Data collection}

For each patient included in this study, we collected the following data at the onset of sepsis or SAE: (1) General data: including age, gender, underlying disease; (2) Data at ICU admission: Acute Physiology and Chronic Health Evaluation II (APACHE II) score, Sequential Organ Failure Assessment score (SOFA score), site of infection, haematological findings (white blood cell (WBC) count, platelet (PLT) count, haematocrit (HCT), procalcitonin (PCT), and serum creatinine $(\mathrm{Cr})$ levels), aetiological information, and outcome indexes (length of stay in the hospital, 28-day mortality).

\section{Assessment of risk of bias}

Two authors independently assessed the risk of bias for each case using the following information outlined: whether the identification of each case of sepsis or SAE was the joint decision of more than three members of the research team; and whether there was evidence of selective reporting of outcomes. Any disagreement was resolved by discussion or by involving a third assessor.

\section{Statistical analysis}

All data were analysed using SPSS 22.0 (SPSS, Inc., NY, USA). Categorical and continuous variables are given as numbers (percentages) and medians [25th-75th percentiles], respectively, and were compared via MannWhitney U tests, $\mathrm{X} 2$ tests, or Fisher's exact tests, as appropriate. SAE- and survival-associated risk factors were identified via multivariate logistic regression analysis. Kaplan-Meier curves were analysed by the log-rank test. $P<0.05$ was the significance threshold.

\section{Results}

Baseline characteristics

After excluding 106 patients, a total of 291 patients with sepsis were retrospectively screened and assigned to the SAE group and non-SAE group. According to the outcome of 28-day mortality, the SAE patients were stratified into survival and non-survival groups (Fig. 1).

Of these 291 sepsis patients, 127 (43.6\%; 90 males) were diagnosed with SAE. The median ages of the SAE and non-SAE groups were $64[48,76]$ and 55[43, 68] years old, respectively, with SAE patients being significantly older than non-SAE patients $(P<0.01)$. We also found significant differences between these two groups with respect to the SOFA score, APACHE II score, and proportion of underlying diseases such as hypertension $(P<0.05$; Table 1$)$.

The primary outcome measures that were monitored in the present study included length of stay (LOS) in the hospital and 28-day mortality. The results showed no significant differences in hospital LOS between the SAE and non-SAE groups. However, we found that the 28day mortality of SAE patients was significantly higher than that of non-SAE patients $(42.5 \%$ vs. $12.8 \%$, $P<0.01$; Table 1).

A Kaplan-Meier survival analysis further confirmed that an SAE diagnosis was associated with significantly poorer 28-day survival in sepsis patients $(H R=3.890$, 95\% CI: 2.035-7.437; $P<$ 0.001; Fig. 2).

\section{Comparison of clinical and etiological findings between SAE and non-SAE patients}

A total of 291 patients with sepsis were admitted to the hospital due to medical disease, emergency surgery or elective surgery. There were no significant differences in the disease types of the patients $(P=0.358)$. The main infection sites were the respiratory tract, biliary tract, gastrointestinal tract, urinary tract, bloodstream, skin and soft tissue. The main pathogens detected were Staphylococcus, Escherichia coli, Enterococcus, Acinetobacter baumannii, Pseudomonas aeruginosa, Klebsiella pneumoniae, and fungi (Candida). We found that patients in the SAE group had significantly higher rates of gastrointestinal infections than non-SAE patients $(26.8 \%$ vs. $15.2 \%, P=0.015)$. In addition, these SAE patients had a higher rate of detection of Enterococcus than non-SAE patients $(16.5 \%$ vs. $7.9 \%, P=0.023)$. No significant differences were observed between groups with respect to other pathogens or infection sites $(P>0.05$; Table 2). Furthermore, these SAE patients had higher serum PCT concentrations $(2.33[1.14,6.97]$ vs. $1.39[0.42,3.99]$, $P<0.01)$ and lower platelet counts $(105[56.0,180.0]$ vs. 168 [110.0, 246.5], $P<0.01)$ than did their non-SAE counterparts (Table 2). 


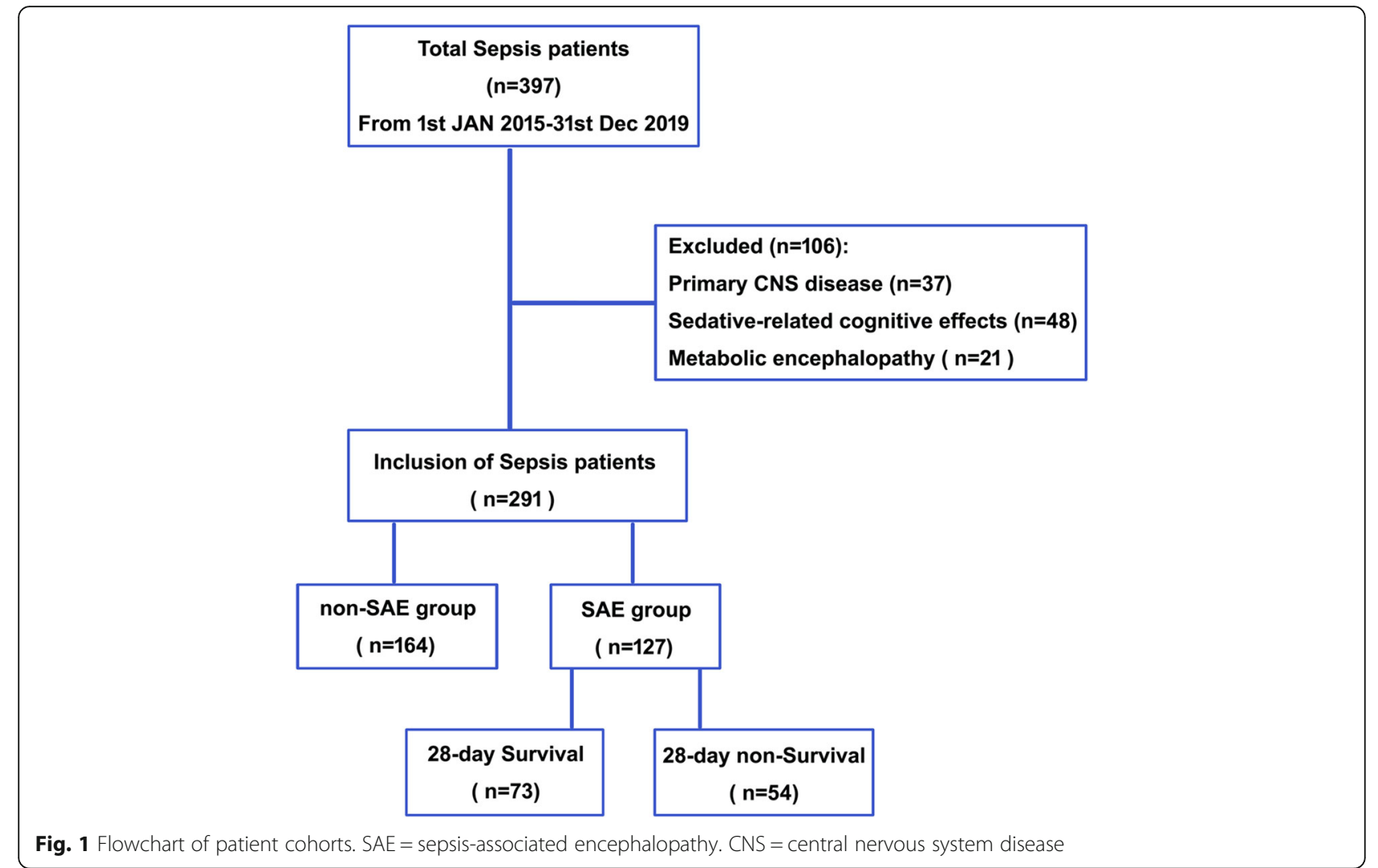

Multivariate analysis of SAE risk factors in sepsis patients Based on the above bivariate analysis, after adjusting the baseline characteristics, infection site, patient type, clinical and aetiological findings of sepsis patients, a subsequent multivariate analysis revealed that both SOFA score (OR per 1-score increment: 1.421, 95\% CI: 1.2441.623, $P<0.001$ ) and APACHE II score (OR per 1 -score increment: 1.239 , 95\% CI: $1.144-1.341, P<0.001)$ were independent risk factors for SAE in ICU patients with sepsis (Table 3).

\section{Multivariate analysis of poor outcome-related factors in SAE patients}

Based upon the outcome of 28-day mortality, 127 SAE patients were divided into a survival group and a nonsurvival group. A multivariate analysis revealed that age (OR per 1-year increment: 1.059, 95\% CI: 1.027 1.093, $P<0.001)$, SOFA score (OR per 1-score increment: 1.167, 95\% CI: $1.009 \sim 1.349, P=0.037)$, and APACHE II score (OR per 1-score increment: 1.178, 95\% CI: 1.063 $1.305, P<0.01)$ were independently associated with 28 day mortality in SAE patients (Table 4).

\section{Discussion}

The occurrence of SAE is one of the main manifestations of organ dysfunction caused by sepsis, excluding clinical or laboratory evidence of a central nervous system infection, a structural abnormality, or another encephalopathy (such as hepatic encephalopathy or uraemic encephalopathy); SAE refers to a diffuse brain dysfunction resulting from sepsis and is mainly exhibited as delirium, cognitive impairment, decreased learning and memory ability, coma, twitch and so on $[3,6]$. The mechanism may involve the dysfunction of cerebral microvascular cells, the loss of blood-brain barrier integrity, mitochondria dysfunction, the activation of microglia and astrocytes, and neuronal death [7, 8]. Currently, the diagnostic criteria and potential risk factors for SAE remain incompletely understood, with no reliable means of clinically evaluating sepsis-associated neurological dysfunction [9]. In the present study, subjective evaluations (performed by clinical staff) and objective indicators (GCS < 15, CAM-ICU for delirium) were combined as the inclusion criteria of SAE patients, and we applied comprehensive exclusion criteria to exclude disturbances due to central nervous system infection, cerebrovascular disease, metabolic disease, sedative-related cognitive effects, toxicosis, etc. More objective indexes should be used in future studies to identify SAE accurately, including craniocerebral ultrasound and bedside electroencephalogram (EEG) monitoring, to detect SAE as early as possible by evaluating cerebral blood flow velocity and changes in EEG activity, especially to predict delirium in critical sepsis patients [4]. The typical changes of 
Table 1 Patients' baseline characteristics

\begin{tabular}{|c|c|c|c|c|}
\hline & All patients $n=291$ & $\begin{array}{l}\text { SAE } \\
n=127\end{array}$ & $\begin{array}{l}\text { non-SAE } \\
n=164\end{array}$ & $P$ \\
\hline Age (years), median & $59[45,72]$ & $64[48,76]$ & $55[43,68]$ & $<0.01^{*}$ \\
\hline \multicolumn{5}{|l|}{ Sex $(\%)$} \\
\hline Male & $213(73.2)$ & $90(70.9)$ & $123(75.0)$ & \multirow[t]{2}{*}{0.430} \\
\hline Female & $78(26.8)$ & $37(29.1)$ & $41(25.0)$ & \\
\hline \multicolumn{5}{|l|}{ Underlying diseases (\%) } \\
\hline Hypertension & $75(25.8)$ & $41(32.3)$ & $34(20.7)$ & $0.025^{*}$ \\
\hline Diabetes & $40(13.7)$ & $23(18.1)$ & $17(10.4)$ & 0.057 \\
\hline COPD & $28(9.6)$ & $10(7.9)$ & $18(11.0)$ & 0.374 \\
\hline $\mathrm{CHF}$ & $37(12.7)$ & $19(15.0)$ & $18(11.0)$ & 0.312 \\
\hline $\mathrm{CHD}$ & $12(4.1)$ & $4(3.1)$ & $8(4.9)$ & 0.462 \\
\hline CKD & $17(5.8)$ & $7(5.5)$ & $10(6.1)$ & 0.833 \\
\hline Malignant tumor & $44(15.1)$ & $22(17.3)$ & $22(13.4)$ & 0.356 \\
\hline Immune diseases & $4(1.4)$ & $1(0.8)$ & $3(1.8)$ & 0.803 \\
\hline Others & $26(8.9)$ & $13(10.2)$ & $13(7.9)$ & 0.493 \\
\hline \multicolumn{5}{|l|}{ Disease severity (median) } \\
\hline SOFA score & $5[2,7]$ & $7[5,10]$ & $3[1,5]$ & $<0.01^{*}$ \\
\hline APACHE II score & $11[8,15]$ & $15[12,19.5]$ & $9[6,11]$ & $<0.01^{*}$ \\
\hline \multicolumn{5}{|l|}{ Outcome } \\
\hline Hospital LOS (median) & $20[11,30]$ & $19[9,28]$ & $21[13,36]$ & 0.143 \\
\hline 28-day mortality (\%) & 77 (26.5) & $54(42.5)$ & $21(12.8)$ & $<0.01^{* *}$ \\
\hline
\end{tabular}

SAE sepsis-associated encephalopathy, COPD chronic obstructive pulmonary disease, CHF chronic heart failure, CHD chronic hepatic disease, CKD chronic kidney disease, SOFA sequential organ failure assessment, APACHE II acute physiology, age and chronic health evaluation II, LOS length of stay

*Statistical analysis using Mann Whitney test, ${ }^{*}$ Statistical analysis using Fisher's exact probability method

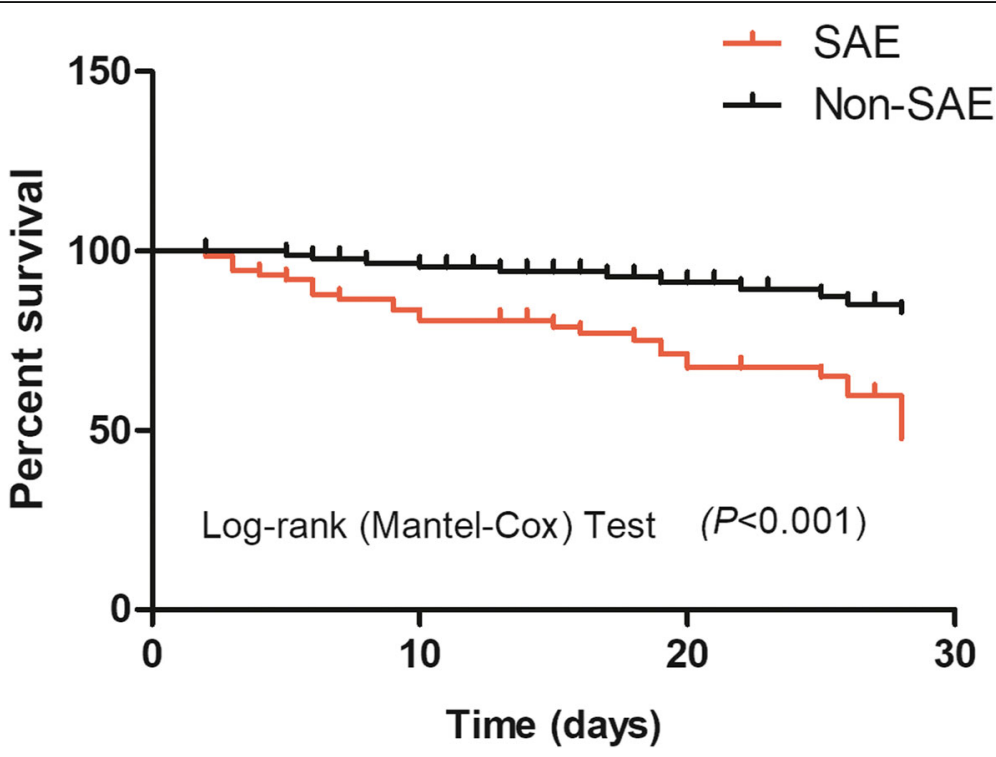

Fig. 2 Kaplan-Meier survival analysis of sepsis patients over 28 days. The SAE group had a significantly poorer 28-day survival than non-SAE group $(\mathrm{HR}=3.890,95 \% \mathrm{Cl}: 2.035 \sim 7.437 ; \mathrm{P}<0.001) . \mathrm{SAE}=$ sepsis-associated encephalopathy 
Table 2 Comparison of clinical and etiological findings in sepsis patients

\begin{tabular}{|c|c|c|c|c|}
\hline & $\begin{array}{l}\text { All patients } \\
\mathrm{n}=\mathbf{2 9 1}\end{array}$ & $\begin{array}{l}\text { SAE } \\
n=127\end{array}$ & $\begin{array}{l}\text { non-SAE } \\
n=164\end{array}$ & $P$ \\
\hline \multicolumn{5}{|l|}{ Disease type $n(\%)$} \\
\hline Medical disease & $198(68.0)$ & $88(69.3)$ & $110(67.1)$ & \multirow[t]{3}{*}{0.358} \\
\hline Emergency surgery & $47(16.2)$ & $23(18.1)$ & $24(14.6)$ & \\
\hline Elective surgery & $46(15.8)$ & $16(12.6)$ & $30(18.3)$ & \\
\hline \multicolumn{5}{|l|}{ Infection sites $n(\%)$} \\
\hline Respiratory tract & $159(54.6)$ & $76(59.8)$ & $83(50.6)$ & 0.076 \\
\hline Biliary tract & $25(8.6)$ & $8(6.3)$ & $17(10.4)$ & 0.220 \\
\hline Gastrointestinal tract & $59(20.3)$ & $34(26.8)$ & $25(15.2)$ & $0.015^{* *}$ \\
\hline Urinary tract & $16(5.5)$ & $9(7.1)$ & $7(4.3)$ & 0.296 \\
\hline Bloodstream & $14(4.8)$ & $7(5.5)$ & $7(4.3)$ & 0.623 \\
\hline Skin and soft tissue & $15(5.2)$ & $5(3.9)$ & $10(6.1)$ & 0.408 \\
\hline \multicolumn{5}{|c|}{ Detected pathogens $n(\%)$} \\
\hline Staphylococcus & $35(12.3)$ & $16(12.6)$ & 19 (11.6) & 0.792 \\
\hline Enterococcus & $34(15.5)$ & $21(16.5)$ & $13(7.9)$ & $0.023^{*}$ \\
\hline Escherichia coli & $67(30.6)$ & $31(24.4)$ & $36(21.9)$ & 0.621 \\
\hline Acinetobacter & $61(20.9)$ & $27(21.3)$ & $34(20.7)$ & 0.913 \\
\hline Pseudomonas & $18(6.2)$ & $8(6.3)$ & $10(6.1)$ & 0.944 \\
\hline Klebsiella & $27(9.3)$ & $11(8.7)$ & $16(9.8)$ & 0.750 \\
\hline Fungus & $32(10.9)$ & $13(10.2)$ & 19 (11.6) & 0.715 \\
\hline Others & $15(5.2)$ & $6(4.7)$ & $9(5.5)$ & 0.770 \\
\hline \multicolumn{5}{|c|}{ Biochemical Indexes (median) } \\
\hline $\mathrm{PCT}(\mu \mathrm{g} / \mathrm{L})$ & $1.89[0.66,5.37]$ & $2.33[1.14,6.97]$ & $1.39[0.42,3.99]$ & $<0.01^{*}$ \\
\hline Endotoxin (EU/mL) & $0.11[0.05,0.20]$ & $0.12[0.05,0.21]$ & $0.10[0.05,0.19]$ & 0.464 \\
\hline WBC $\left(\times 10^{9} / \mathrm{L}\right)$ & $10.8[7.60,15.8]$ & $10.9[8.5,16.2]$ & $11.35[7.70,15.88]$ & 0.245 \\
\hline $\operatorname{PLT}\left(\times 10^{9} / \mathrm{L}\right)$ & $152[76.7,191.5]$ & $105[56.0,180.0]$ & $168[110.0,246.5]$ & $<0.01^{*}$ \\
\hline $\mathrm{HCT}$ & $30.1[27.0,33.0]$ & $20.3[18.1,22.1]$ & $33.4[33.0,34.3]$ & 0.069 \\
\hline $\mathrm{Cr}(\mu \mathrm{mol} / \mathrm{L})$ & $78[52.0,95.8]$ & $78[59.0,109.8]$ & $73.5[51.5,87.0]$ & 0.315 \\
\hline
\end{tabular}

SAE sepsis-associated encephalopathy, PCT procalcitonin, WBC white blood cell, PLT blood platelet, $H C T$ hematocrit, $C r$ serum creatinine

*Statistical analysis using Mann Whitney test, **Statistical analysis using Fisher's exact probability method

EEG in SAE patients include an excessive $\theta$ rhythm, an increased $\delta$ rhythm, a three-phase wave and burst suppression [10]. In addition, SAE patients exhibit increased brain injury biomarkers (i.e., neuron-specific enolase, S-100 betaprotein) and neuroradiological abnormalities [11-13].

In recent decades, sepsis and SAE have been the focus of intensive medicine research, with high morbidity and mortality. The recently reported 28-day mortality and 180-day mortality rates of SAE were 45.95 and $55.41 \%$,

Table 3 Multivariate analysis of risk factors in Sepsis-Associated Encephalopathy patients

\begin{tabular}{llll}
\hline Value & OR & $\mathbf{9 5 \% ~ C l}$ & $\boldsymbol{P}$ \\
\hline APACHE II (per 1-score increment) & 1.239 & $1.144 \sim 1.341$ & $<0.001$ \\
SOFA (per 1-score increment) & 1.421 & $1.244 \sim 1.623$ & $<0.001$ \\
\hline
\end{tabular}

SOFA sequential organ failure assessment, APACHE II acute physiology, age and chronic health evaluation II, LOS length of stay respectively [2]. In the present study, $43.6 \%$ of patients with sepsis were identified as having SAE, exhibiting a $42.5 \%$ of 28 -day mortality, which was significantly higher than that of non-SAE patients. However, there was no significant difference in the hospitalization time, suggesting that encephalopathy resulting from sepsis may have a greater influence on long-term outcomes, including longterm cognitive dysfunction and functional disability, which

Table 4 Multivariate analysis of 28-day mortality in SepsisAssociated Encephalopathy patients

\begin{tabular}{llll}
\hline Value & OR & $\mathbf{9 5 \% ~ C I}$ & $\boldsymbol{P}$ \\
\hline Age (per 1-year increment) & 1.059 & $1.027 \sim 1.093$ & $<0.001$ \\
SOFA score (per 1-score increment) & 1.167 & $1.009 \sim 1.349$ & 0.037 \\
APACHE II score (per 1-score increment) & 1.178 & $1.063 \sim 1.305$ & $<0.01$
\end{tabular}

SOFA sequential organ failure assessment, APACHE II acute physiology, age and chronic health evaluation II 
seriously decrease the quality of life and bring a great burden to patients, their families and even society [14]. In addition, it should be noted that the choice of hospital LOS as an outcome indicator may result in statistical bias. The shortening of LOS may be due to the critical condition of patients and who succumbed during early stages/ initial phase of resuscitation, rather than the result of effective treatment.

Elderly patients exhibit a higher risk and mortality from sepsis [15]. In the present study, we also found the age was an independent risk factor for 28-day mortality of SAE patients. In addition, severe patients with underlying diseases often progress more rapidly and have poor prognosis. Especially when the underlying diseases are hypertension, diabetes, acute renal injury, and chronic obstructive pulmonary disease (COPD), such patients might be more likely to develop central nervous system complications [2, 16-18]. We found that patients with sepsis who had underlying hypertension had a higher risk of progression to SAE. Moreover, we found that the SOFA score and APACHE II score were not only independent risk factors for SAE in sepsis patients but also independent risk factors for 28-day mortality in SAE patients. This further confirms the advantages of SOFA scores and APAC HE II scores in assessing the severity and prognosis of critical patients $[19,20]$ and suggests that the underlying health status and severity of sepsis patients are closely related to the occurrence of encephalopathy and adverse outcomes. A significantly decreased platelet count in SAE patients was also found in the present study, which suggested that platelets participate in immune and inflammatory responses against various pathogens, apart from their important role in the coagulation process [21].

It is interesting in the present study that the incidence of gastrointestinal infection and the rate of detection of $\mathrm{En}$ terococcus were increased significantly in sepsis patients with encephalopathy, which suggests that the gut or gut microbiota might participate in the pathogenesis of SAE. The reported studies have shown that changes in gut homeostasis and the gut microbiome could influence the physiology, behaviour and cognitive function related to the brain, and the interaction between gut microbiota and the brain has gradually become a hot topic in neuroscience; this interaction may ensure the maintenance of gut homeostasis but also exhibit multiple effects on emotion, motivation and cognitive function $[22,23]$. This complex interaction between the gut and brain has been named the "gut-brain axis (GBA)", which plays an important role in monitoring and integrating intestinal function and linking the emotional and cognitive centres of the brain with peripheral intestinal functions and mechanisms [24]. It has been confirmed that the gut microbiome is involved in the pathophysiological development process of neurodegenerative diseases, stroke, emotional and affective disorders and other central nervous system diseases or encephalopathy [25-27]. Patients with sepsis that is directly influenced by gastrointestinal infection or antibiotic administration [28] may develop disturbances of the gut microbiota. Animal experiments have found that faecal bacteria transplantation can change the gut microbiota of septic mice, suggesting that faecal bacteria transplantation and vagal nerve block are potential therapeutic targets in SAE [29]. The effects of gut microbiota disturbance and the GBA on SAE development deserve further study, especially with respect to upstream and downstream initiation mechanisms, which may provide more ideas to explore the pathogenesis and early diagnosis of SAE.

\section{Limitations}

In summary, in the present retrospective study, we obtained some results that provide certain guiding significance for clinical practice. However, there are still some limitations. First, the relatively small sample size of this single-centre retrospective study and the lower freedom of statistical processing could have impaired the statistical analysis. In addition, we mainly relied on subjective evaluation and exclusive diagnosis to identify SAE. Future studies should add more objective indexes, such as craniocerebral ultrasound and bedside EEG monitoring, to help us identify SAE more accurately and in a timely manner.

\section{Conclusion}

In the present retrospective study, we found that nearly half of patients with sepsis developed SAE, which was closely related to poor outcomes. The identified risk factors for SAE included older age, underlying hypertension, gastrointestinal infection, enterococcal infection, and decreased platelet count. Both the SOFA score and APACHE II score were independent risk factors for predicting the occurrence and adverse outcome of SAE. Future studies should be focused on investigating the longterm prognosis and cognitive function of SAE patients and the upstream initiation mechanism of SAE.

\section{Supplementary information}

Supplementary information accompanies this paper at https://doi.org/10. 1186/s12873-020-00374-3.

\section{Additional file 1.}

\section{Abbreviations}

SAE: Sepsis-associated encephalopathy; SOFA: Sequential Organ Failure Assessment; APACHE II: Acute Physiology and Chronic Health Evaluation II; GCS: Glasgow coma score; CAM-ICU: Confusion Assessment Method for the Intensive Care Unit; ICU: Intensive Care Unit; WBC: White blood cell; PLT: Platelet; HCT: Haematocrit; PCT: Procalcitonin; Cr: Serum creatinine; LOS: Length of stay; COPD: Chronic obstructive pulmonary disease; GBA: Gutbrain axis; EEG: Electroencephalogram. 


\section{Authors' contributions}

$J C, X S, G J$, and SX contributed to the design and data collection of this work. $J C, X S, G J$, and SX participated in the preparation of the manuscript. JC, XS, $G J, Y Z, W H$, and SX contributed to the revision of the manuscript. MD contributed to the EEG data exhibition in author's responses to the reviewer's comments. All authors approved the final version of the manuscript.

\section{Funding}

This research received funding support from the Zhejiang Provincial Natural Science Foundation of China (Grant No. LY19H030007). The funding body had no role in the design, collection, analysis, interpretation of data, or writing of the manuscript.

\section{Availability of data and materials}

The datasets used and/or analysed during the current study are available from the corresponding author on reasonable request.

\section{Ethics approval and consent to participate}

The study was approved by the ethics committee of Affiliated Hangzhou First People's Hospital, Zhejiang University School of Medicine. The institutional Ethics and Research Committee waived the requirement for consent because the project is a retrospective analysis of routinely collected administrative data.

\section{Consent for publication}

Not applicable.

\section{Competing interests}

The authors declare no conflict of interest.

\section{Author details}

'Department of Critical Care Medicine, Affiliated Hangzhou First People's Hospital, Zhejiang University School of Medicine, No.261, Huansha Road, Zhejiang 310006, Hangzhou, China. ${ }^{2}$ Department of Radiology, Affiliated Hangzhou First People's Hospital, Zhejiang University School of Medicine, Zhejiang, Hangzhou, China.

Received: 3 July 2020 Accepted: 28 September 2020

Published online: 06 October 2020

\section{References}

1. Fu Q, Wu J, Zhou XY, Ji MH, Mao QH. Li Q2, et al. NLRP3/Caspase-1 pathway-induced Pyroptosis mediated cognitive deficits in a mouse model of Sepsis-associated encephalopathy. Inflammation. 2019;42(1):306-18. https://doi.org/10.1007/s10753-018-0894-4.

2. Sonneville R, de Montmollin E, Poujade J, Garrouste-Orgeas M, Souweine B, Darmon $\mathrm{M}$, et al. Potentially modifiable factors contributing to sepsisassociated encephalopathy. Intensive Care Med. 2017;43(8):1075-84. https://doi.org/10.1007/s00134-017-4807-z.

3. Gofton TE, Young GB. Sepsis-associated encephalopathy. Nat Rev Neurol. 2012;8(10):557-66. https://doi.org/10.1038/nrneurol.2012.183.

4. Feng Q, Ai YH, Gong H, Wu L, Ai ML, Deng SY, et al. Characterization of Sepsis and Sepsis-associated encephalopathy. I Intensive Care Med. 2019; 34(11-12):938-45. https://doi.org/10.1177/0885066617719750.

5. Rhodes A, Evans LE, Alhazzani W, Levy MM, Antonelli M, Ferrer R, et al. Surviving Sepsis campaign: international guidelines for Management of Sepsis and Septic Shock: 2016. Intensive Care Med. 2017;43(3):304-77. https://doi.org/10.1007/s00134-017-4683-6.

6. Zampieri FG, Park M, Machado FS, Azevedo LC. Sepsis-associated encephalopathy: not just delirium. Clinics (Sao Paulo). 2011;66(10):1825-31. https://doi.org/10.1590/s1807-59322011001000024.

7. Haileselassie B, Joshi AU, Minhas PS, Mukherjee R, Andreasson KI, Mochly-Rosen D. Mitochondrial dysfunction mediated through dynamin-related protein 1 (Drp1) propagates impairment in blood brain barrier in septic encephalopathy. J Neuroinflammation. 2020;17(1):36. https://doi.org/10.1186/s12974-019-1689-8.

8. Ren C, Yao RQ, Zhang H, Feng YW, Yao YM. Sepsis-associated encephalopathy: a vicious cycle of immunosuppression. J Neuroinflammation. 2020;17(1):14. https:/doi.org/10.1186/s12974-020-1701-3.

9. Sonneville R, Verdonk F, Rauturier C, Klein IF, Wolff M, Annane D, et al. Understanding brain dysfunction in sepsis. Ann Intensive Care. 2013;3(1):15. https://doi.org/10.1186/2110-5820-3-15.
10. Kuperberg SJ, Wadgaonkar R. Sepsis-associated encephalopathy: the bloodbrain barrier and the Sphingolipid rheostat. Front Immunol. 2017;8:597. https://doi.org/10.3389/fimmu.2017.00597.

11. Yao B, Zhang LN, Ai YH, Liu ZY, Huang L. Serum S100ß is a better biomarker than neuron-specific enolase for sepsis-associated encephalopathy and determining its prognosis: a prospective and observational study. Neurochem Res. 2014;39(7):1263-9. https://doi.org/10.1007/s11064-014-1308-0.

12. González-García S, González-Quevedo A, Fernández-Concepción O, PeñaSánchez M, Menéndez-Saínz C, Hernández-Díaz Z, et al. Short-term prognostic value of serum neuron specific enolase and S100B in acute stroke patients. Clin Biochem. 2012;45(16-17):1302-7. https://doi.org/10.1016/j.clinbiochem.

13. Stubbs DJ, Yamamoto AK, Menon DK. Imaging in sepsis-associated encephalopathy insights and opportunities. Nat Rev Neurol. 2013;9(10):55161. https://doi.org/10.1038/nrneurol.2013.177.

14. Pandharipande PP, Girard TD, Jackson JC, Morandi A, Thompson JL, Pun BT, et al. Long-term cognitive impairment after critical illness. N Engl J Med. 2013;369(14):1306-16. https://doi.org/10.1056/NEJMoa1301372.

15. Martin GS, Mannino DM, Moss M. The effect of age on the development and outcome of adult sepsis. Crit Care Med. 2006;34(1):15-21. https://doi. org/10.1097/01.ccm.0000194535.82812.ba.

16. Hajjar I, Keown M, Frost B. Antihypertensive agents for aging patients who are at risk for cognitive dysfunction. Curr Hypertens Rep. 2005;7(6):466-73. https://doi.org/10.1007/s11906-005-0043-y.

17. Sonneville R, Vanhorebeek I, den Hertog HM, Chrétien F, Annane D, Sharshar T, et al. Critical illness-induced dysglycemia and the brain. Intensive Care Med. 2015:41(2):192-202. https://doi.org/10.1007/s00134-014-3577-0.

18. Siew ED, Fissell WH, Tripp CM, Blume JD, Wilson MD, Clark AJ, et al. Acute kidney injury as a risk factor for delirium and coma during critical illness. Am J Respir Crit Care Med. 2017;195(12):1597-607. https://doi.org/10.1164/ rccm.201603-04760C.

19. Matsuda J, Kato S, Yano H, Nitta G, Kono T, Ikenouchi T, et al. The Sequential Organ Failure Assessment (SOFA) score predicts mortality and neurological outcome in patients with post-cardiac arrest syndrome. J Cardiol. 2020;(20):30107-6. https://doi.org/10.1016/j.jjcc.2020.03.007.

20. Martin-Loeches I, Guia MC, Vallecoccia MS, Suarez D, Ibarz M, Irazabal M, et al. Risk factors for mortality in elderly and very elderly critically ill patients with sepsis: a prospective, observational, multicenter cohort study. Ann Intensive Care. 2019;9(1):26. https://doi.org/10.1186/s13613-019-0495-X.

21. Hottz ED, Bozza FA, Bozza PT. Platelets in Immune Response to Virus and Immunopathology of Viral Infections. Front Med (Lausanne). 2018;5:121. https://doi.org/10.3389/fmed.2018.00121.

22. Schmidt C. Mental health: thinking from the gut. Nature. 2015;518(7540): S12-5. https://doi.org/10.1038/518S13a.

23. Smith PA. The tantalizing links between gut microbes and the brain. Nature. 2015;526(7573):312-4. https://doi.org/10.1038/526312a.

24. Montiel-Castro AJ, González-Cervantes RM, Bravo-Ruiseco G, Pacheco-López G. The microbiota-gut-brain axis: neurobehavioral correlates, health and sociality. Front Integr Neurosci. 2013;7:70. https://doi.org/10.3389/fnint.2013.00070.

25. Friedland RP. Mechanisms of molecular mimicry involving the microbiota in neurodegeneration. J Alzheimers Dis. 2015;45(2):349-62. https://doi.org/10. 3233/jad-142841.

26. Stanley D, Mason L, Mackin KE, Srikhanta YN, Lyras D, Prakash MD, et al. Translocation and dissemination of commensal bacteria in post-stroke infection. Nat Med. 2016;22(11):1277-84. https://doi.org/10.1038/nm.4194.

27. Park AJ, Collins J, Blennerhassett PA, Ghia JE, Verdu EF, Bercik P, et al. Altered colonic function and microbiota profile in a mouse model of chronic depression. Neurogastroenterol Motil. 2013;25(9):733-e575. https://doi.org/10.1111/nmo.12153.

28. De Waele JJ, Lipman J, Akova M, Bassetti M, Dimopoulos G, Kaukonen M, et al. Risk factors for target non-attainment during empirical treatment with B-lactam antibiotics in critically ill patients. Intensive Care Med. 2014;40(9): 1340-51. https://doi.org/10.1007/s00134-014-3403-8.

29. Li S, LV J, Li J, Zhao Z, Guo H, Zhang Y, et al. Intestinal microbiota impact sepsis associated encephalopathy via the vagus nerve. Neurosci Lett. 2018; 662:98-104. https://doi.org/10.1016/j.neulet.2017.10.008.

\section{Publisher's Note}

Springer Nature remains neutral with regard to jurisdictional claims in published maps and institutional affiliations. 